\title{
Methane line opacities in very cool stellar objects
}

\author{
P. H. Hauschildt ${ }^{1}$, R. Warmbier ${ }^{2}$, R. Schneider ${ }^{2}$, and T. Barman ${ }^{3}$ \\ 1 Hamburger Sternwarte, Gojenbergsweg 112, 21029 Hamburg, Germany \\ e-mail: yeti@hs.uni-hamburg.de \\ 2 Max-Planck-Institut für Plasmaphysik, Wendelsteinstr. 1, 17491 Greifswald, Germany \\ 3 Lowell Observatory, Flagstaff, AZ, USA
}

Received 22 April 2009 / Accepted 26 June 2009

\begin{abstract}
Aims. We investigate the effects of different line data for methane ${ }^{12} \mathrm{CH}_{4}$ on the structures of model atmospheres and low resolution synthetic spectra for ultra-cool substellar objects.

Methods. For each set of methane line data we compare the resulting model atmospheres and spectra computed with the general purpose model atmosphere code PHOENIX.

Results. The new HGW methane line data compares well to the HITRAN2004 data. We find the the HITRAN2004 methane lines are in some bands stronger than the HGW lines, resulting in deeper absorption bands in the synthetic spectra. Conclusions.
\end{abstract}

Key words. molecular data - stars: atmospheres - stars: low-mass, brown dwarfs

\section{Introduction}

The discovery of ultra-cool substellar objects has lead to the definition of the new spectral class T. These objects are also called "methane dwarfs" as one of their defining characteristics are strong absorptions bands of methane in the IR spectra. Modeling their atmospheres and spectra, therefore, requires complete and detailed spectral line data for methane.

In Warmbier et al. (2008, hereafter Paper I), we described a new line list for methane that was computed with the intent to provide a complete line list with relatively low accuracy for individual lines but good accuracy for the overall opacity in order to accurately model the effect of the methane lines on the structure of the atmosphere and on low-resolution synthetic spectra. This is justified due to a number of reasons: a) the exact details of the individual line's position is not important for the structure of the atmosphere to the level of a few angstrøm shift in line position, b) the details of individual lines are washed out in low resolution $(\lambda / \Delta \Lambda \leq 2000)$ spectra. On the other hand, the line list has to be as complete as possible in order to correctly describe the temperature variation of the integrated line opacity throughout the atmosphere, where temperatures range from below $1000 \mathrm{~K}$ to $10^{4} \mathrm{~K}$. Such a line list may even be useful to model moderately high resolution spectra as observable molecular spectral features in T dwarfs are typically formed by the overlap of many broadened spectral lines, where the main line broadening mechanisms are pressure broadening and (rapid) rotation of the object.

In this paper, we compare model atmosphere structures and synthetic spectra for a number of different sources of methane lines to the line list discussed in Paper I. The goal is to quantify the differences in the structures and the synthetic spectra for a number of different methane line databases. This will help to establish confidence limits on the sensitivity of the atmospheric structure and the synthetic spectra to variations in the line input data. We will compare the results for these methane line lists:

1. the list discussed in Paper I Warmbier et al. (2008), hereafter: HGW. This list contains 1219509 methane lines;

2. the methane data available in HITRAN2004 Rothman et al. (2004), hereafter: HITRAN2004. This list contains 143602 methane lines;

3. methane data from the GEISA database (Husson et al. 1992), hereafter: GEISA, with 37075 lines.

The line data and numbers are only for the main isotope of methane, ${ }^{12} \mathrm{CH}_{4}$. Data for the other trace isotopes of methane were always taken from HITRAN2004 for all models and spectra shown in this paper.

\section{Method}

In this paper we compare the model structures and synthetic spectra computed using different line databases for methane. In order to keep the comparison valid, all other relevant model data, e.g., abundances, equation of state, and line data for other molecules, will be identical for all models and spectra presented here.

\subsection{Model atmospheres}

We use version 16 of the general purpose model atmosphere code PHOENIX (Hauschildt \& Baron 1999) to compute the model atmospheres and synthetic spectra. This version of PHOENIX includes a number of improvements compared to previous versions that were used by, e.g., Maness et al. (2007); Seifahrt et al. (2007); Johnas et al. (2007a); Lançon et al. (2007).

1. A complete new equation of state for ions, molecules and condensation (ACES; Barman, in preparation). ACES uses 
modern thermodynamic data and can provide the partial pressures of several 100 species down to temperatures of $100 \mathrm{~K}$ while being substantially faster than the old equation of state. Details and comparisons to other results will be presented in Barman \& Hauschildt (in preparation).

2. Updated opacity databases, including HITRAN2004 (Rothman et al. 2004) and metal hydrides (e.g., Bernath 2006, and references therein).

3. Improved line profiles for atomic lines that affect the structure of the atmospheres (Johnas et al. 2007a,b; Allard et al. 2003).

All models considered here were iterated to convergence (typical errors in the flux are less that $1 \%$ and temperature changes substantially smaller than $1 \mathrm{~K}$ ) for their given setup if not specified otherwise. The low resolution synthetic spectra are taken directly from the results of the structure iterations. The abundances are taken from Asplund et al. (2005) if not stated otherwise. The condensation of dust particles is approximately treated in chemical equilibrium and the dust particles are assumed to have completely gravitationally settled below the atmosphere, resulting in the "COND" setup of Allard et al. (2001). The latter approximation appears to be reasonable for $T_{\text {eff }}<1000 \mathrm{~K}$, in these model the convection zone starts deeper below the outermost layers than in the $\mathrm{L}$ and $\mathrm{M}$ dwarfs and thus the dust has time to sink below the radiative zone into a convection zone that starts at relatively large optical depths. However, for effective temperatures larger than about $1000 \mathrm{~K}$ more realistic DRIFTPHOENIX/ACES models (Witte et al. 2009) should be used as non-phase-equilibrium dust clouds form and have a significant impact on the structure of the atmosphere and the resulting spectra. However, these models are not yet generally available and we concentrate here on the differences between different methane line lists rather than model realism.

\subsection{Results}

\section{3. model parameters}

We have calculated a number of models with different parameters appropriate for the regime of T dwarfs: the gravity is fixed at $\log (g)=5.0$ and the effective temperatures range from $600 \mathrm{~K}$ to $1500 \mathrm{~K}$. In the following we compare the results for models converged with their respective setups if not indicated otherwise.

\section{4. model structures}

In Fig. 1 we show 4 model atmosphere structures computed for the 3 different methane line lists. All models were fully converged with their setup and only the choice of the methane line data is different for the models. The differences between the structures appear to be rather small, in order to show the differences more clearly we plot the temperature differences in Fig. 2 as function of the optical depth at $1.2 \mu \mathrm{m}$ in the continuum with the HGW model structure as the (arbitrary) baseline. The temperature differences between the HGW model and the HITRAN2004 model are below $\approx 50 \mathrm{~K}$ up to an optical depth, $\tau_{\text {std }}$, of about unity, The differences to the GEISA models are comparable but have a different sign at low optical depths. This indicates that the integrated methane opacity for the HGW and HITRAN2004 methane lines is different, with the HITRAN2004 data giving the larger integrated opacity. This causes stronger cooling at small optical depth and a larger back-warming at larger depths. The HGW and HITRAN2004 line lists differ strongly in line density.

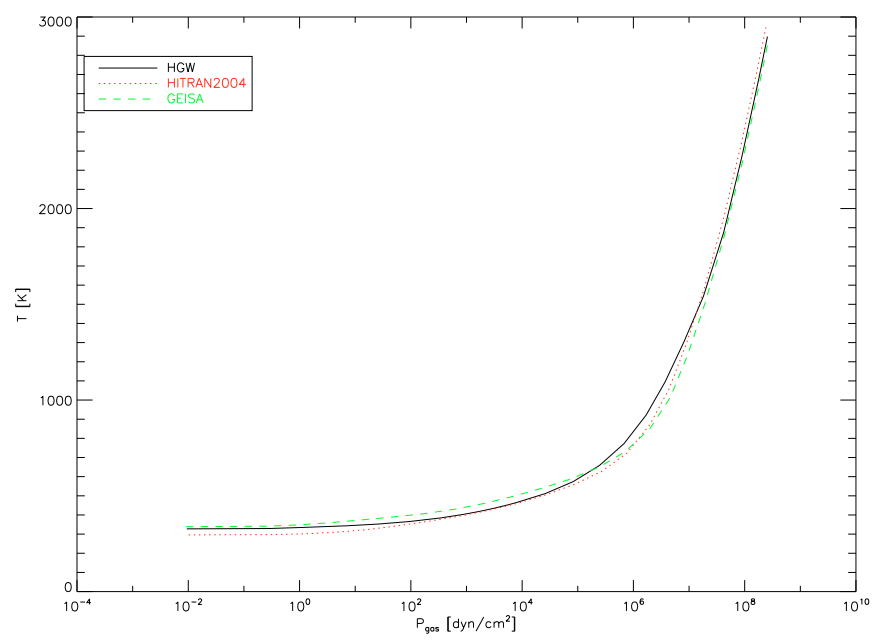

Fig. 1. Atmospheric structures for $T_{\text {eff }}=1000 \mathrm{~K}, \log (g)=5.0$ calculated with different line lists.

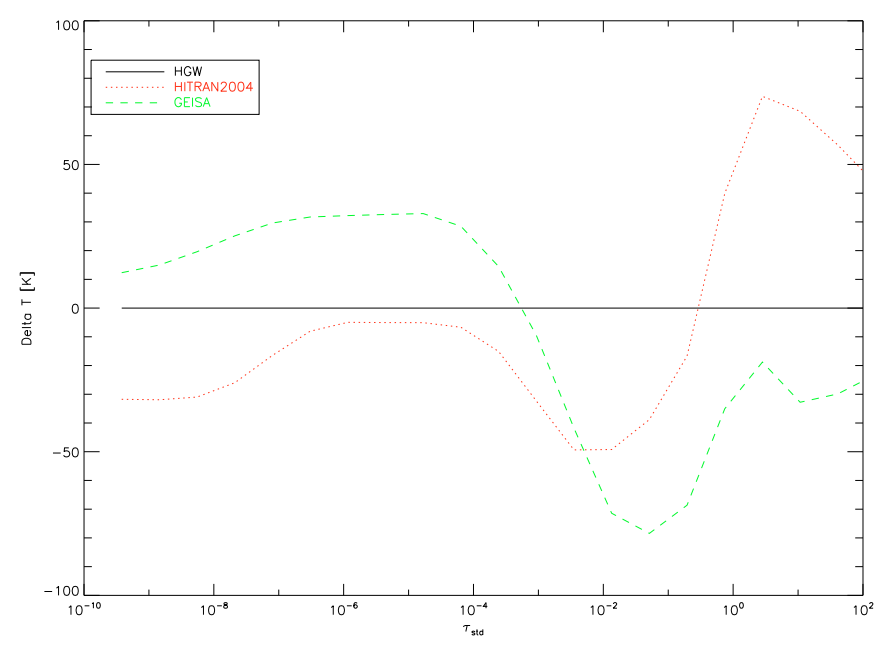

Fig. 2. As Fig. 1, but now the temperature differences are shown as function of gas pressure for the models with $T_{\text {eff }}=1000 \mathrm{~K}$ and $\log (g)=5.0$.

While the HITRAN2004 list has more accurate lines, the HGW line list is much more complete. This leads to relatively higher accuracy of calculations at low pressures for HITRAN2004, while the HGW line list should produce better accuracy for medium to high pressure.

\section{5. low resolution synthetic spectra}

We compare the low resolution IR spectra of 4 models in Fig. 3 for $T_{\text {eff }}=1000 \mathrm{~K}$, in Fig. 4 for $T_{\text {eff }}=1500 \mathrm{~K}$, and in Fig. 5 for $T_{\text {eff }}=600 \mathrm{~K}$. The spectra were computed at a step size of $2 \AA$ and smoothed with a boxcar kernel of $40 \AA$, to result in a resolution of $\lambda / \Delta \lambda \approx 750$ at $3 \mu$. The figure shows clearly that at this resolution the HGW and HITRAN2004 spectra are similar, with the exception of the region around $2.3 \mu$ where the HITRAN2004 spectrum shows significantly less flux (with corresponds to overall larger methane opacity) than the HGW spectrum. In the region between $\approx 3 \mu \mathrm{m}$ and $\approx 4.5 \mu \mathrm{m}$ a similar but smaller effect can be seen. Here, the differences are smaller for lower effective temperatures. The GEISA spectra show much weaker methane features than the other two line databases. For temperatures between $500 \mathrm{~K}$ and $800 \mathrm{~K}$ the HGW and HITRAN2004 


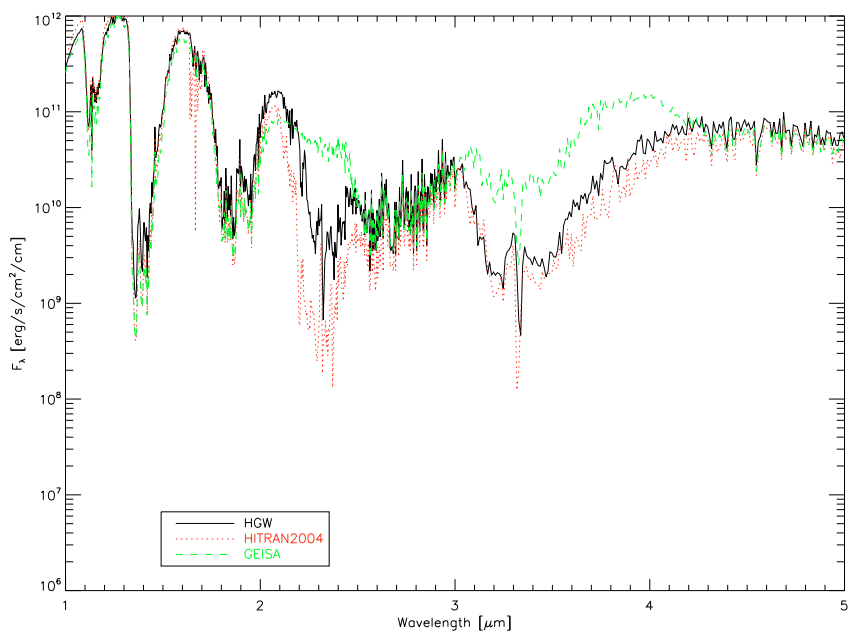

Fig. 3. Low resolution (40 A) synthetic IR spectra computed for the models with $T_{\text {eff }}=1000 \mathrm{~K}$ and $\log (g)=5.0$.

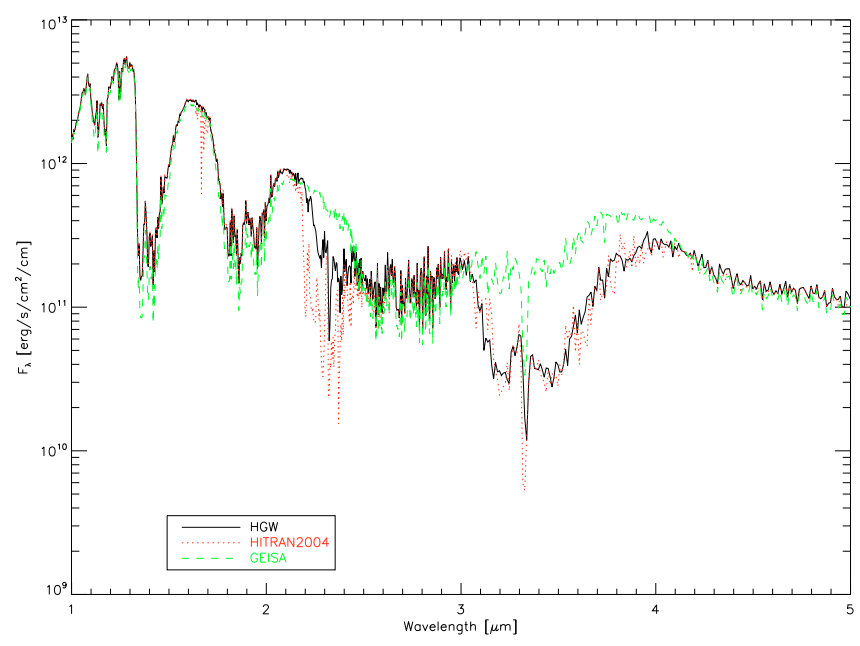

Fig. 4. Low resolution $(40 \AA)$ synthetic IR spectra computed for the models with $T_{\text {eff }}=1500 \mathrm{~K}$ and $\log (g)=5.0$.

databases should be of similar quality. While a moderate underestimation of methane opacity is true for the HGW line list at all temperatures, the $2.3 \mu$ region seems to be overestimated by the HITRAN2004 line list. We expect the HGW list to be complete for this temperature range (99HITRAN2004 has only sparse lines in this region. This may lead to an artificial redistribution of intensity to these lines, causing wrong spectral features.

The region centered around $2.3 \mu$ for $T_{\text {eff }}=1000 \mathrm{~K}$ is enlarged in Fig. 6. Figure 7 shows that the differences are mostly due to a few stronger individual features in the HITRAN2004 methane spectra that are not as strong in the HGW data, e.g., the very strong HITRAN2004-feature at about $2.2 \mu$. These stronger features then cause the low-resolution spectra to be significantly different. In addition, the average line strength in the HITRAN2004 data is somewhat larger than in the HGW dataset. This becomes clear in Fig. 8 which shows the same region as before but now computed at a resolution of $0.05 \AA$. This is not an effect of the different structures as similar effects are seen if the structure is artificially kept fixed as discussed in Paper I.

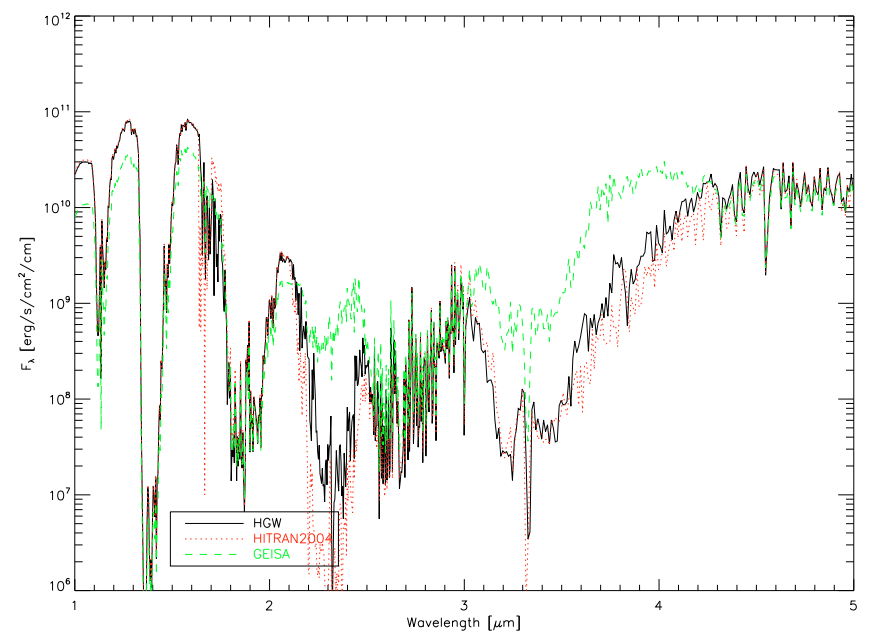

Fig. 5. Low resolution (40 $\AA$ ) synthetic IR spectra for $T_{\text {eff }}=600 \mathrm{~K}$ and $\log (g)=5.0$.

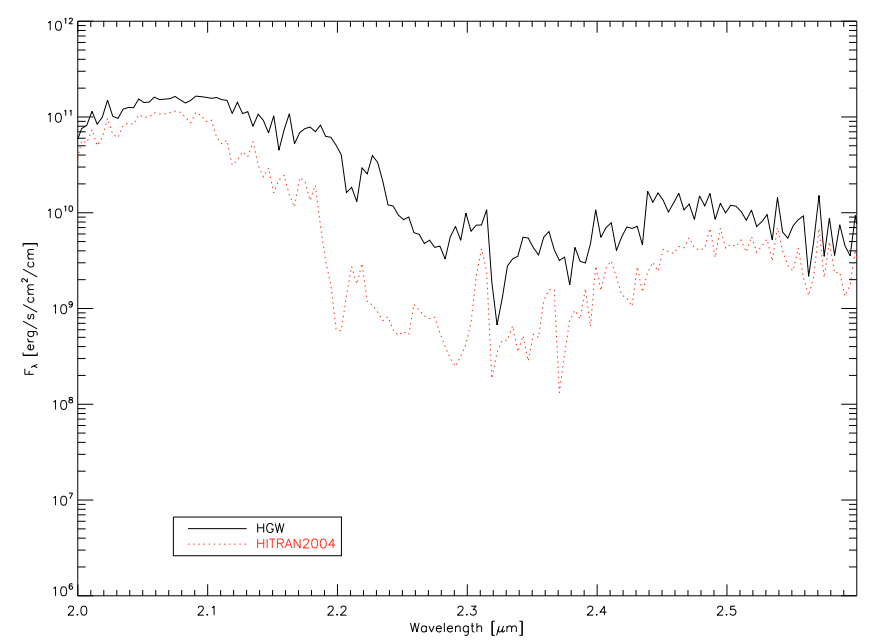

Fig. 6. Low resolution ( $40 \AA$ ) synthetic IR spectra for $T_{\text {eff }}=1000 \mathrm{~K}$ and $\log (g)=5.0$. The plot highlights the differences between HITRAN2004 and HGW in the range around $2.3 \mu \mathrm{m}$ where the HITRAN2004 lines are substantially stronger than the HGW lines.

\section{6. comparison to observed spectra}

We compare the low resolution synthetic spectra for the HGW and the HITRAN2004 line lists to observed T8 dwarf spectra of McLean et al. (2003) in Figs. 9 and 10. This is not intended to be a optimal fit to the observations, but to illustrate the possibility of observable differences between the two line lists. Therefore, the models were not tuned to fit the observations optimally, we just selected the best spectra from the available models, allowing for parameter differences between the models for different methane lists. In the figures the differences due to the different data for methane are only detectable above $2.2 \mu$, the differences between $1.6 \mu$ and $1.8 \mu$ are not well fit and cannot be used to decide empirically between the two line lists. In both cases, the HITRAN2004 data result in a best fit with an effective temperature $200 \mathrm{~K}$ to $300 \mathrm{~K}$ higher than the best fit with the HGW methane lines, which is a very large systematic error due to variations in fundamental molecular data. This difference is not surprising, as the HITRAN2004 lines are stronger and the fits were 


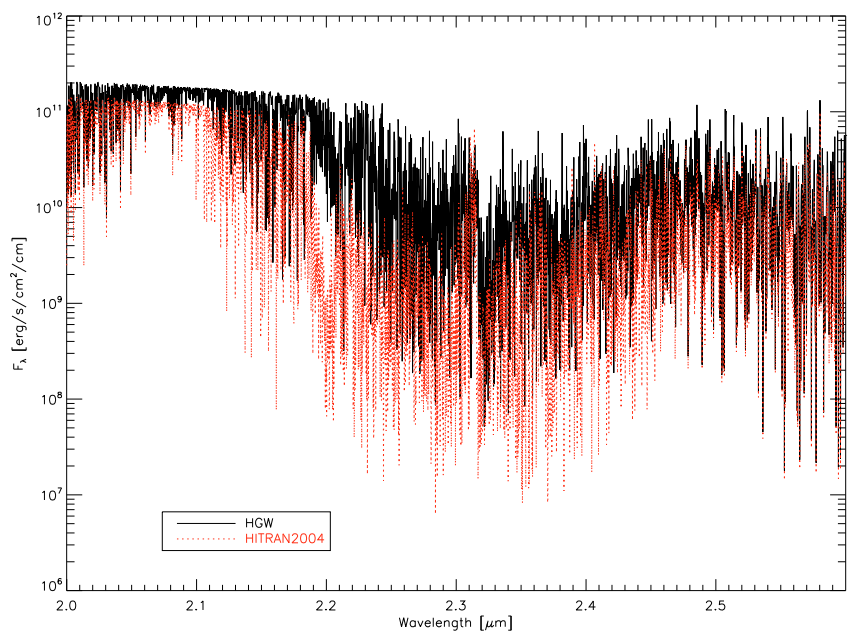

Fig. 7. Low resolution ( $2 \AA$ ) IR spectra for $T_{\text {eff }}=1000 \mathrm{~K}$.

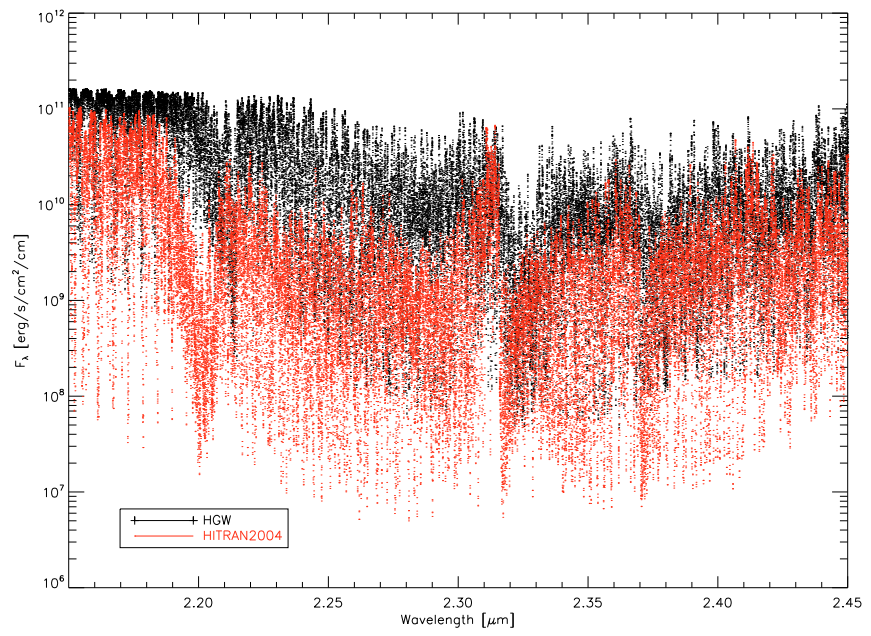

Fig. 8. High resolution $(0.05 \AA)$ IR spectra for $T_{\text {eff }}=1000 \mathrm{~K}$.

primarily based on the methane bands in the spectrum. Outside the methane bands the spectra are too similar to conclusively decide which methane dataset gives the overall best fit. The bands around $1.6 \mu \mathrm{m}$ indicate a significantly lower temperature that is not supported by the other features in the observed spectra. It appears that there are either missing/too weak methane bands, another missing opacity source or clouds effects are missing in the simple models. In order to obtain a meaningful empirical differences the observations need to cover larger wavelengths and reach a reasonable signal to noise ratio also at low flux levels, as the contrast between peaks and valleys in the broad band features is significantly different between the different line data.

\section{Summary and conclusions}

We have compared the structures and synthetic spectra of model atmospheres computed with different line list data for ${ }^{12} \mathrm{CH}_{4}$. The new HGW methane line data compares well to the HITRAN2004 data. We find the the HITRAN2004 methane lines are in some bands stronger than the HGW lines, resulting in deeper absorption bands in the synthetic spectra. This leads to significant differences in effective temperatures derived from synthetic spectra in the NIR band for late T dwarfs, indicating the

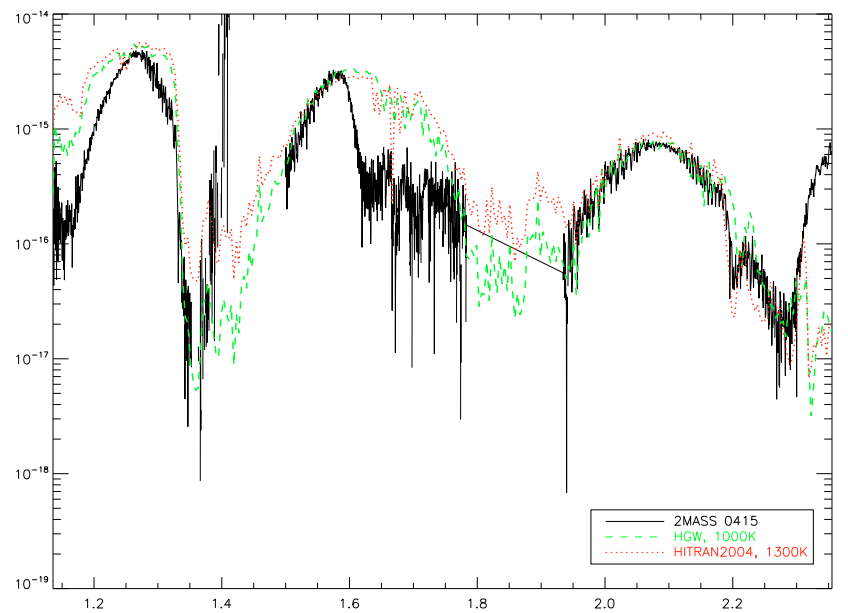

Fig. 9. The T8 dwarf 2MASS 0415-09 compared to models with $T_{\text {eff }}=$ $1000 \mathrm{~K}$ (HGW methane data) and $T_{\mathrm{eff}}=1300 \mathrm{~K}$ (HITRAN2004 methane data), $\log (g)=5.0$ and solar abundances.

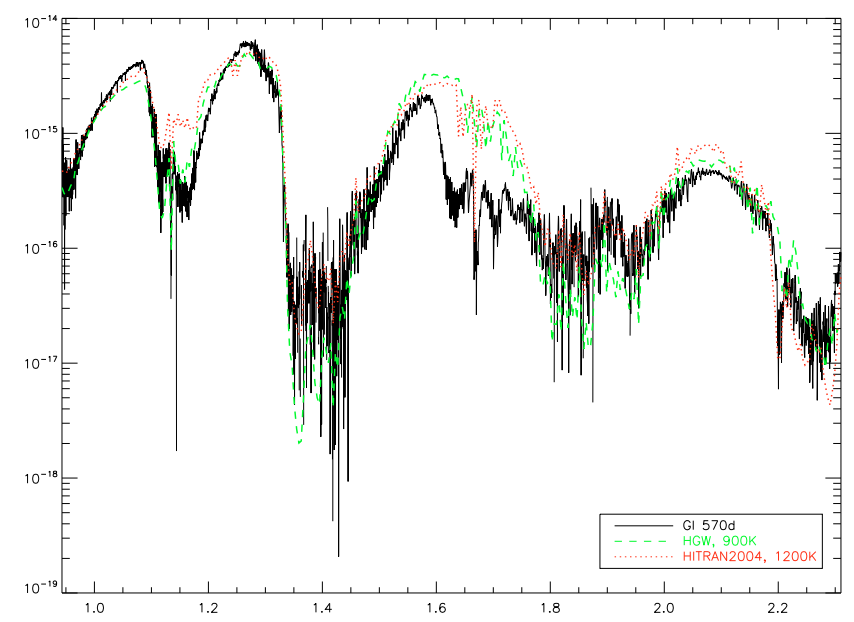

Fig. 10. The T8 dwarfGl 570d compared to models with $T_{\text {eff }}=900 \mathrm{~K}$ (HGW methane data) and $T_{\text {eff }}=1200 \mathrm{~K}$ (HITRAN2004 methane data), $\log (g)=5.0$ and solar abundances.

importance of the methane bands for parameter determinations of T dwarfs and the still very large intrinsic systematic errors due to fundamental molecular data uncertainties. The differences in determined effective temperatures will also have effects on estimates of cloud physics in cool substellar objects, as the properties of the clouds and the feedback with the atmosphere depend on the effective temperature and the gravities of the model atmospheres.

Acknowledgements. This work was supported in part DFG GrK 1351. Some of the calculations presented here were performed at the Höchstleistungs Rechenzentrum Nord (HLRN); at the NASA's Advanced Supercomputing Division's Project Columbia, at the Hamburger Sternwarte Apple G5 and Delta Opteron clusters financially supported by the DFG and the State of Hamburg; and at the National Energy Research Supercomputer Center (NERSC), which is supported by the Office of Science of the US Department of Energy under Contract No. DE-AC03-76SF00098. We thank all these institutions for a generous allocation of computer time. 


\section{References}

Allard, F., Hauschildt, P. H., Alexander, D. R., Tamanai, A., \& Schweitzer, A. 2001, ApJ, 556, 357

Allard, N. F., Allard, F., Hauschildt, P. H., Kielkopf, J. F., \& Machin, L. 2003, A\&A, 411, L473

Asplund, M., Grevesse, N., \& Sauval, A. J. 2005, in Cosmic Abundances as Records of Stellar Evolution and Nucleosynthesis, ed. T. G. Barnes, III, \& F. N. Bash, ASP Conf. Ser., 336, 25

Bernath, P. 2006, in Astrochemistry - From Laboratory Studies to Astronomical Observations, ed. R. I. Kaiser, P. Bernath, Y. Osamura, S. Petrie, \& A. M. Mebel, ASP Conf. Ser., 855, 143
Hauschildt, P. H., \& Baron, E. 1999, Journal of Computational and Applied Mathematics, 109, 41

Husson, N., Bonnet, B., Scott, N., \& A. C. 1992, JQSRT, 48, 509

Johnas, C. M. S., Hauschildt, P. H., Schweitzer, A., et al. 2007a, A\&A, 466, 323 Johnas, C. M. S., Hauschildt, P. H., Schweitzer, A., et al. 2007b, A\&A, 475, 1039

Lançon, A., Hauschildt, P. H., Ladjal, D., \& Mouhcine, M. 2007, A\&A, 468, 205 Maness, H. L., Marcy, G. W., Ford, E. B., et al. 2007, PASP, 119, 90

McLean, I. S., McGovern, M. R., Burgasser, A. J., et al. 2003, ApJ, 596, 561

Rothman, L., Jacquemart, D., Barbe, A., et al. 2004, JQSRT, 96, 139

Seifahrt, A., Neuhäuser, R., \& Hauschildt, P. H. 2007, A\&A, 463, 309

Warmbier, R., Schneider, R., Hauschildt, P., et al. 2008, A\&A, 495, 655

Witte, S., Helling, C., \& Hauschildt, P. 2009, A\&A, submitted 